\title{
Maximum Throughput Analysis and Enhancement of Slotted ALOHA for Multihop Ad Hoc Networks
}

\author{
Zhongbang Yao \\ Department of Electronic Engineering \\ Tsinghua University \\ Beijing, China \\ Email: zbyao@eee.hku.hk
}

\author{
Victor O. K. Li \\ Department of Electrical and \\ Electronic Engineering \\ The University of Hong Kong \\ Hong Kong, China \\ Email: vli@eee.hku.hk
}

\author{
Zhigang Cao \\ Department of Electronic Engineering \\ Tsinghua University \\ Beijing, China \\ Email: czg-dee@mail.tsinghua.edu.cn
}

\begin{abstract}
In this paper, we analyze the maximum throughputs of slotted-ALOHA-based multihop ad hoc networks with and without capture, by considering the degree (number of neighbors) of each node, and, different from prior research, allowing each node to have a different transmission probability. We propose a novel enhanced slotted ALOHA scheme, in which each station adaptively transmits packets according to the degrees of the stations' neighbors. The analytical and simulation results show that the enhanced scheme can improve the network performance greatly.
\end{abstract}

\section{INTRODUCTION}

In a multihop ad hoc network, the stations (i.e., terminals, or nodes) can communicate with each other without any stationary infrastructure. The packets may collide at a receiver due to its receiving multiple packets simultaneously [5], hence degrading the network performance irrespective of the media access control (MAC) protocol adopted in the network [3]-[5], [9].

From prior research [1]-[3], [6]-[9], the network performance (e.g., throughput or delay) is greatly affected by the network node density. In these efforts, the authors always assume that all the stations transmit packets with the same predefined constant probability $p$, and then investigate the network performance under different values of $p$. In some recent research analyzing carrier sense multiple access with collision avoidance (CSMA/CA) [9]-[12], again a similar assumption is adopted. However, in practical network environment, the node density is dynamic when the stations move and thus is not known to the stations, making it difficult to properly determine $p$ so as to maximize specific network metrics. Thus, although prior research shows the relationship between the throughput, packet transmission probability, and node density, it is not readily translated into a practical protocol since it is difficult to get the correct node density. Even if the stations know the average node density of the whole network, the success or failure of a packet transmission is mostly determined by the local node density, not by the average node density of the whole network.

Define the degree of a node as the number of nodes reachable within a single hop. Intuitively, if there are more neighbors, i.e., higher node degree, the station should transmit less to avoid packet collisions. As far as the authors know, prior research has not revealed the relationship between the packet transmission probability and the degrees of neighboring stations to maximize specific network metrics.

In this paper, we investigate the relationship between the throughput, packet transmission probability, and the degrees of neighboring stations. Based on this relationship, each station can adaptively tune its local packet transmission probability. We use the instantaneous and average one-hop throughput as the network metrics.

The rest of this paper is organized as follows. Section II presents the network model. We analyze the one-hop throughput of slotted ALOHA with and without capture in a multihop network in Section III. Based on the analysis, an enhanced slotted ALOHA scheme is proposed in this section. We give our conclusions in Section IV.

\section{Network Model}

In this paper, we make the following assumptions.

- Network Topology: We assume a quasi-static network topology. The stations are distributed in an area according to the two-dimensional Poisson distribution with parameter $\lambda$. During a specific period, one or more time slots, the network topology is fixed. Then we generate another network topology according to the same two-dimensional Poisson distribution to simulate the random movements of stations.

- Stations: Each station can transmit and receive in a circular area with radius $R$. Every station knows its degree and the degree of each of its neighbors. In Section III-C, we will describe how the stations learn such information. Different from prior research, which always assumes that the stations transmit packets with a fixed probability $p$, we assume the packet transmission probability is a function of node degrees, i.e., $p=$ $p\left(N_{s}, N_{s_{1}}, \ldots, N_{s_{N_{s}-1}}, N_{d}, N_{d_{1}}, \ldots, N_{d_{N_{d}-1}}\right)$, in which $N_{s}$ is the degree of the source $s, N_{d}$ is the degree of the destination $d, N_{s_{i}}$ is the degree of neighbor $i\left(1 \leq i \leq N_{s}-1\right)$ of $s$, and $N_{d_{i}}$ is degree of neighbor $i\left(i \leq i \leq N_{d}-1\right)$ of $d$. 
- MAC Protocol: Each station accesses the wireless channel according to the slotted ALOHA protocol with or without capture, in which a slot time period accounts for the propagation delay, one packet transmission delay, and station processing time. The packet size is fixed. Packet loss is only caused by packet collisions. We ignore wireless channel errors which may also cause packet losses.

- Traffic: There is always a packet in the station buffer waiting to be transmitted, i.e., the network is heavily-loaded. But we do not consider the effect of acknowledgement packets.

These assumptions are typical in prior research [1], [2], [9] except for the packet transmission probability function $p$. Based on these assumptions, the average degree of a station can be calculated as $N=\lambda \pi R^{2}$.

In this paper, we define the instantaneous one-hop throughput as the packet successful transmission probability of one specific slot, i.e., throughput conditioning on the degree of each station in the network being fixed. While the average one-hop throughput is defined as the statistical average of instantaneous one-hop throughput, i.e., the instantaneous onehop throughput un-conditioning on the specific slots. In the following sections, we use the following notations.

$X_{i}^{s}$ : the event that the degree of station $s$ is $i$, where the neighbors are indexed with $s_{1}, s_{2}, \ldots, s_{i}$, respectively.

$s \rightarrow d$ : the event that the packet transmission is successful from station $s$ to station $d$.

$p_{i}$ : the packet transmission probability of station $i$.

$T_{n c i}, T_{c i}$ : the instantaneous one-hop throughput for noncapture and capture slotted ALOHA.

$T_{n c a}, T_{c a}$ : the average one-hop throughput for non-capture and capture slotted ALOHA.

\section{Performance Analysis of Slotted AlOHA}

\section{A. Slotted ALOHA without Capture}

As described above, the station transmission probability is a function of the the node degree and the degrees of the node's neighbors. Assume that the degree of the source $s$ is $N_{s}$, the degree of the destination $d$ is $N_{d}$. The transmission from $s$ to $d$ is shown in Figure 1, in which $s_{i}$ is the neighbor of $s$, and $d_{i}$ is the neighbor of $d$. From this figure, we can get

$$
\begin{aligned}
T_{n c i}= & P_{r}\left\{s \rightarrow d \mid X_{N_{s}}^{s}, X_{N_{s_{1}}}^{s_{1}}, \ldots, X_{N_{s_{N_{s}-1}}}^{s_{N_{s}-1}}, X_{N_{d}}^{d},\right. \\
& \left.X_{N_{d_{1}}}^{d_{1}}, \ldots, X_{N_{d_{N_{d}-1}}}^{d_{N_{2}-1}}\right\} \\
= & p_{s}\left(1-p_{d}\right) \prod_{i=1}^{N_{d}-1}\left[1-p_{d_{i}}\right]
\end{aligned}
$$

where $p_{s}=p_{s}\left\{N_{s}, N_{s_{1}}, \ldots, N_{s_{N_{s}-1}}, N_{d}, N_{d_{1}}, \ldots, N_{d_{N_{d}-1}}\right\}$ denotes the packet transmission probability of station $s$; $p_{d}=p_{d}\left\{N_{d}, N_{d_{1}}, \ldots, N_{d_{N_{d}-1}}, N_{d^{\prime}}, N_{d^{\prime} 1}, \ldots, N_{d^{\prime} N_{d^{\prime}}-1}\right\}$ denotes the packet transmission probability of station $d$, in which $d^{\prime}$ is the destination of station $d$, and $N_{d^{\prime}}$ is the degree of $d^{\prime} ; p_{d_{i}}$ denotes the packet transmission probability of station

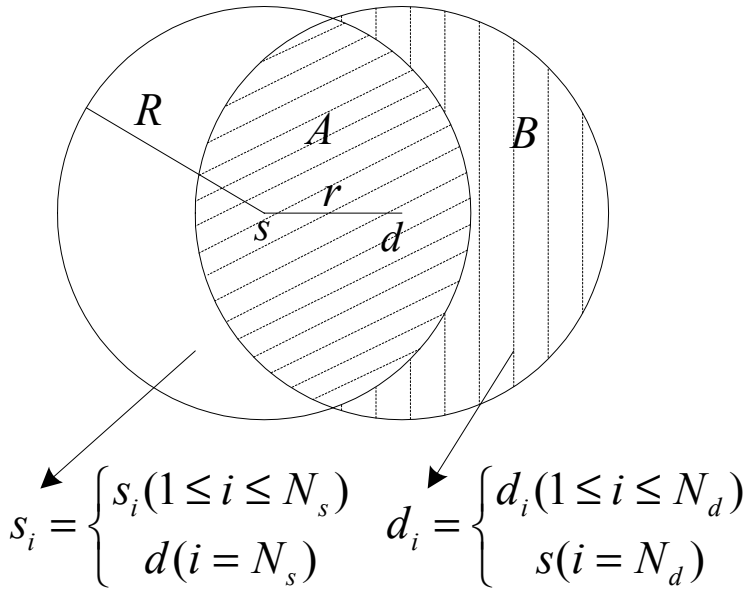

Fig. 1. Illustration of the common area between the source and the destination without capture

$d_{i}\left(1 \leq i \leq N_{d}-1\right)$, which is also a function of the number of neighbors.

For a randomly selected station $r s$, the probability of the number of its neighbors being $i$ can be expressed as follows,

$$
P_{i}^{r s}=P_{r}\left\{X_{i}^{r s}\right\}=\frac{N^{i}}{i !} e^{-N} .
$$

Thus, the average one-hop throughput is,

$$
\begin{aligned}
T_{n c a}= & \left(1-e^{-N}\right) \sum_{N_{d}=0}^{\infty} \sum_{N_{s}=0}^{\infty} \sum_{N_{s_{1}}=0}^{\infty} \ldots \sum_{N_{s_{N_{s}-1}}=0}^{\infty} \sum_{N_{d_{1}}=0}^{\infty} \ldots \\
& \sum_{N_{d_{N_{d}-1}}=0}^{\infty}\left(P_{N_{s}}^{s} P_{N_{d}}^{d} \prod_{i=1}^{N_{s}-1} P_{N_{s_{i}}}^{s_{i}} \prod_{j=1}^{N_{d}-1} P_{N_{d_{j}}}^{d_{j}}\right) T_{n c i}, \quad \text { (3) }
\end{aligned}
$$

where $1-e^{-N}$ is the probability that there exists at least one station in the transmission range of $s$. Clearly, in a real ad hoc network, to maximize $T_{n c i}$ is more practical and easier than to maximize $T_{n c a}$, and in order to implement this, we should carefully design the packet transmission probability $p_{s}, p_{d}$, and $p_{d_{i}}$. However, maximizing equation (1) is at least an $N_{s}+N_{d}$ dimensional optimization problem, which is mathematically intractable. Thus, we simplify this problem by assuming the packet transmission probability is a function of the degree of $d$, and furthermore, we let the packet transmission probabilities of other stations to be the same as that of the source station $s$, i.e., $p_{s}=p_{d}=p_{d_{i}}=p\left(N_{d}\right)$.

The above two simplifications are reasonable because: 1) the most important factor that affects the packet transmission is the numbers of nodes around the source and destination, and these two numbers are closely related because of the common area of the source and destination; 2) the stations are peers in ad hoc networks, which means that the stations should have equal opportunities in transmitting packets. Based on the simplifications, we get the following expressions,

$$
T_{n c i}=p\left(N_{d}\right)\left[1-p\left(N_{d}\right)\right]^{N_{d}},
$$




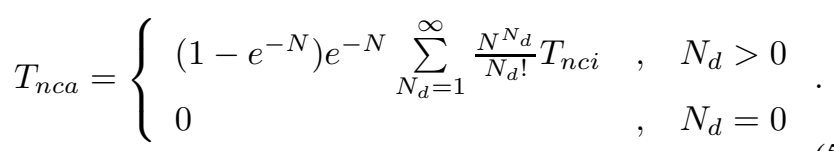

If we let $p\left(N_{d}\right)=p_{c}$ in which $p_{c}$ is a constant value with $0 \leq p_{c} \leq 1$, then we have the following average one-hop throughput,

$T_{n c a}=\left\{\begin{array}{ll}p_{c}\left(1-e^{-N}\right) e^{-N} \sum_{N_{d}=1}^{\infty} \frac{N^{N_{d}}}{N_{d} !}\left(1-p_{c}\right)^{N_{d}} & , \quad N_{d}>0 \\ 0 & , \quad N_{d}=0\end{array}\right.$.

Let $\frac{d T_{n c i}}{d p}=0$, we get

$$
\begin{aligned}
p\left(N_{d}\right)_{o p t} & =\frac{1}{N_{d}+1} \\
& =\frac{1}{2} \text { when } N_{d}=1 \\
& \approx \frac{1}{N_{d}} \text { when } N_{d} \rightarrow \infty .
\end{aligned}
$$

Therefore,

$T_{n c a_{o p t}}=\left(1-e^{-N}\right) e^{-N} \sum_{N_{d}=1}^{\infty} \frac{N^{N_{d}}}{N_{d} !} \frac{1}{N_{d}+1}\left(1-\frac{1}{N_{d}+1}\right)^{N_{d}}$,

for $N_{d}>0 . T_{n c a_{\text {opt }}}$ becomes 0 if $N_{d}=0$. That is, the optimal packet transmission probability corresponds to distributing the transmission probability uniformly among the stations in the covering area of the destination. This result is reasonable, which can be seen from the fact that $p\left(N_{d}\right)=\frac{1}{2}$ when $N_{d}=1$ (i.e., there just exist two stations, the source and destination) and $p\left(N_{d}\right) \approx \frac{1}{N_{d}}$ when $N_{d} \rightarrow \infty$.

Through the above analysis, $p\left(N_{d}\right)_{o p t}$ is a function of $N_{d}$. Actually, as described above, there is a common area between the source and destination as shown in Figure 1, that is, there are some common stations which are the neighbors of both the source and the destination. $N_{s}$ and $N_{d}$ are not independent. If the number of stations in the common area is $N_{c c}\left(N_{s}, N_{d}\right)$, then $N_{d}$ can be re-written as $N_{d}=\left(N_{d}-N_{c c}\left(N_{s}, N_{d}\right)\right)+$ $N_{c c}\left(N_{s}, N_{d}\right)$. This means the optimal packet transmission probability is actually a function of the degrees of the source and destination.

\section{B. Slotted ALOHA with Capture}

In slotted ALOHA with capture (shown in Figure 2), we denote the capture distance to be $r_{c}=\alpha r$, where $r$ is the distance between a source $s$ and its destination $d$, and $\alpha(\alpha \geq 1)$ is called the "capture ratio." In order to make the "optimal solution" mathematically tractable and simple, we use the average values instead of the integration operations in the following analysis. In this paper, we assume that the capture distance is limited to the station transmission range $R$, and the number of stations in the capture distance of a destination station is $N_{A c}$ (i.e., the number of station in area $A_{c}$ is $N_{A c}$ as shown in Figure 2). The average of $N_{A c}$ is denoted as $\overline{N_{A c}}$. In the following, we will first derive the expression of $\overline{N_{A c}}$ and then analyze the one-hop throughput.

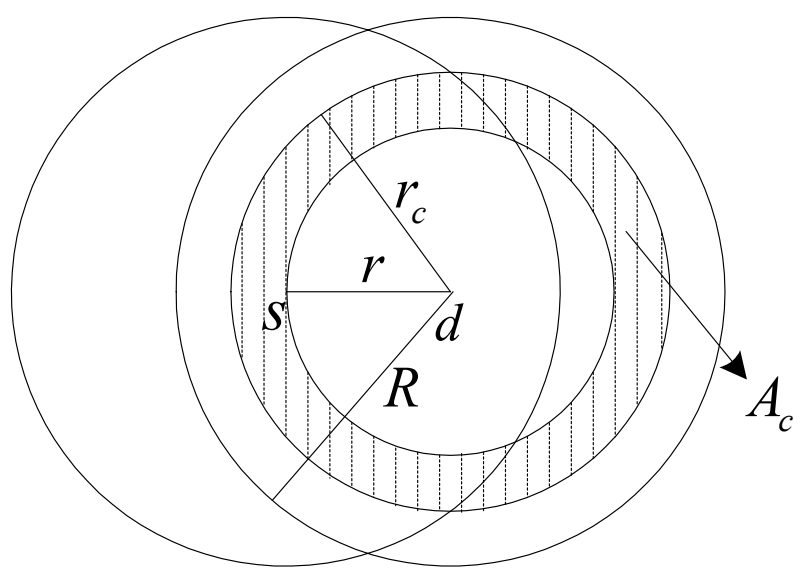

Fig. 2. Illustration of the capture region for node $d$

Let $Y$ be the random variable corresponding to the distance between the source and its destination, then we have

$$
P_{r}\{Y \leq r\}=\frac{\pi r^{2}}{\pi R^{2}},
$$

and the corresponding density function is

$$
f(r)=\frac{2 r}{R^{2}} .
$$

Thus, assuming the degree of the destination is $N_{d}$, we get

$$
\overline{N_{A c}}=N_{d} \int_{r=0}^{R} \frac{\pi r_{c}{ }^{2}}{\pi R^{2}} f(r) d r
$$

Since it is possible that $\alpha r$ is bigger than $R$, and the range that a station transmits or receives is limited to $R$, there are two cases: 1) when $\alpha r \leq R(r \leq R / \alpha), \pi r_{c}{ }^{2}$ becomes $\pi \alpha^{2} r^{2}$; 2) when $\alpha r>R(r>R / \alpha), \pi r_{c}^{2}$ becomes $\pi R^{2}$. Therefore, we obtain

$$
\overline{N_{A c}}=N_{d}\left(1-\frac{1}{2 \alpha^{2}}\right)
$$

Similar to the assumptions used when analyzing the performance of slotted ALOHA without capture, we assume that the packet transmission probability is a function of the degree of the destination. And since $s$ does not know the current value of real $N_{A c}$ in practice, we use $\overline{N_{A c}}$ instead of $N_{A c}$ for a fixed network topology. Thus, we have the instantaneous and average one-hop throughputs as follows,

$$
\begin{gathered}
T_{c i}=p\left(N_{d}\right)\left[1-p\left(N_{d}\right)\right]^{\overline{N_{A c}}}, \\
T_{c a}=\left\{\begin{array}{ll}
\left(1-e^{-N}\right) e^{-N} \sum_{N_{d}=0}^{\infty} \frac{N^{N_{d}}}{N_{d} !} T_{c i} & , \quad N_{d}>0 \\
0 & , \quad N_{d}=0
\end{array} .\right.
\end{gathered}
$$

Similarly, if we let $p\left(N_{d}\right)=p_{c}$, we have the following expression,

$T_{c a}=\left\{\begin{array}{ll}p_{c}\left(1-e^{-N}\right) e^{-N} \sum_{N_{d}=1}^{\infty} \frac{N^{N_{d}}}{N_{d} !}\left(1-p_{c}\right)^{\overline{N_{A c}}} & , \quad N_{d}>0 \\ 0 & , N_{d}=0\end{array}\right.$. 
Then we obtain the optimal packet transmission probability by letting $\frac{d T_{c i}}{d p}=0$,

$$
p\left(N_{d}\right)_{o p t}=\frac{1}{\overline{N_{A c}}+1}
$$

Thus, we have

$T_{c a_{\text {opt }}}=\left(1-e^{-N}\right) e^{-N} \sum_{N_{d}=1}^{\infty} \frac{N^{N_{d}}}{N_{d} !} \frac{1}{\overline{N_{A c}}+1}\left(1-\frac{1}{\overline{N_{A c}}+1}\right)^{\overline{N_{A c}}}$,

for $N_{d}>0$. When $N_{d}=0, T_{c a_{o p t}}=0$.

\section{Enhanced Slotted ALOHA}

From the above analysis, the maximum throughput is a function of the degrees of a station's neighbors. Thus, in order to maximize the throughput, we should let each station know its degree and the degrees of its neighbors. Generally, there are three methods to implement this goal. 1) The degree is broadcast through a separate wireless channel; 2) Each station estimates its degree through monitoring packet transmissions and then broadcast this number; 3) Each station estimates its degree and transmit it in the data or control packet as a degree field. For simplicity, we employ the third method.

As soon as the station receives a packet from $a$ ( $a$ is a randomly selected station), the number of neighbors will be increased by one if no packets have been received from $a$ before. In addition, the station can obtain the degree of $a$ from the degree field of any passing packet originated from $a$. On the other hand, if there are no packets arriving from an existing neighbor $a$ for a predefined period $T_{p}$, then $a$ will be considered to have departed and the degree will be decreased by one.

After getting the degrees, each station can dynamically adjust the local packet transmission according to the optimal packet transmission probability derived above. Thus, the network throughput can be maintained at a relatively high level.

\section{Performance Evaluation}

We run simulations to show the performance of enhanced slotted ALOHA schemes with and without capture. In the simulations, there are 100 nodes, distributed in a $250 \mathrm{~m} \times 250 \mathrm{~m}$ area, leading to an average node density $\lambda=\frac{100}{250 \times 250}=$ $1.6 \times 10^{-3}$. We set $N=\lambda \pi R^{2}=3$, thus each node has a transmission range of about $24.4 \mathrm{~m}$. We repeat each simulation point 30 times and average them. In order to reduce the negative effects induced by the "border effect", i.e., the nodes close to the edges of the simulation area may receive more successful packets because of their smaller degrees, we just consider the nodes located between $\frac{1}{4}$ and $\frac{3}{4}$ of the horizontal and vertical axes, i.e., the nodes in the middle $125 \mathrm{~m} \times 125 \mathrm{~m}$ area. In the non-enhanced schemes (i.e., fixing packet transmission probability $p$ when running a simulation), the topology will be generated every time slot according to the two-dimensional Poisson distribution with parameter $\lambda$. For the enhanced schemes, since the nodes need time to estimate their degrees, the simulation program will generate another

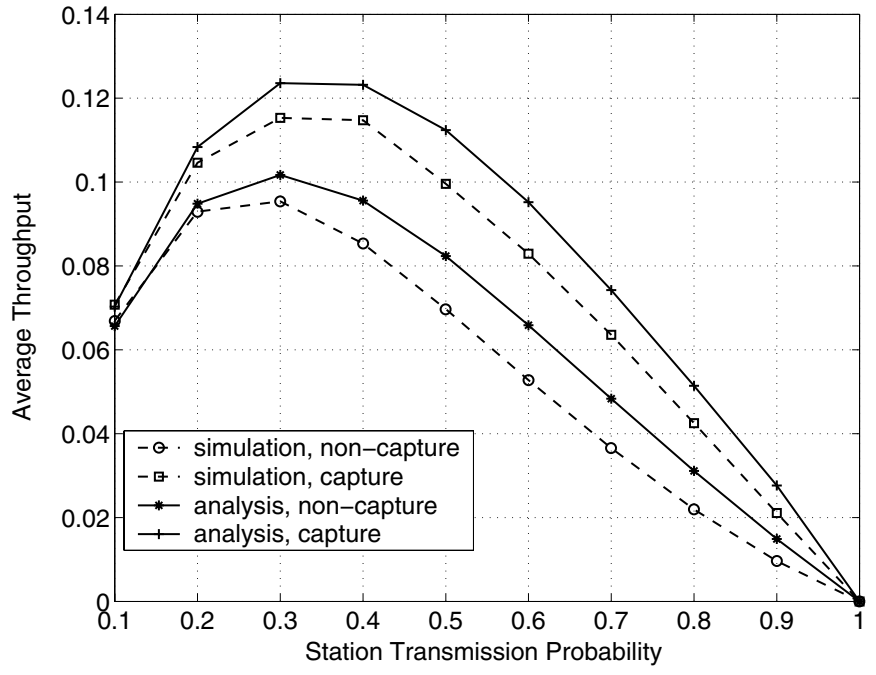

Fig. 3. Average throughput of non-capture and capture slotted ALOHA schemes

TABLE I

AVERAGE NUMBER OF COMPETING STATIONS

\begin{tabular}{|l|l|l|}
\hline & non-capture & capture \\
\hline 0.1 & 3.3496 & 2.6288 \\
\hline 0.2 & 3.3493 & 2.6250 \\
\hline 0.3 & 3.3532 & 2.6236 \\
\hline 0.4 & 3.3526 & 2.6226 \\
\hline 0.5 & 3.3516 & 2.6198 \\
\hline 0.6 & 3.3498 & 2.6182 \\
\hline 0.7 & 3.3506 & 2.6126 \\
\hline 0.8 & 3.3508 & 2.6089 \\
\hline 0.9 & 3.3497 & 2.6028 \\
\hline
\end{tabular}

topology every 500 slots with the same Poisson parameter $\lambda$, and $T_{p}=20$ slots. The $95 \%$ confidence interval of the average values are on the order of $10^{-3}$ to $10^{-4}$, too small to be shown in the following figures.

Figure 3 ( $\alpha=1.5$ for the capture ALOHA scheme) shows the performance of non-capture and capture slotted ALOHA schemes when the station transmission probability is fixed, changing from 0.1 to 1 , together with the analysis results of one-hop average throughput. Clearly, we can see that the simulation results are always smaller than the corresponding analysis results. This is due to the "denser nodes" in the center of the simulation area, which is clearly shown in Table I. For the non-capture slotted ALOHA scheme, the average number of competing stations are greater than the corresponding average node density of 3 for the whole simulation area. Concerning the capture slotted ALOHA, the analysis shows that $\overline{N_{A c}}=3 \times\left(1-\frac{1}{2 \times 1.5^{2}}\right)=2.3333$. However, the average number of competing stations in the middle simulation area is about 2.6. Therefore, due to the greater average numbers of competing stations in the middle simulation area, the simulation results are smaller than the analysis results.

The effect of different capture ratios on the throughput of capture slotted ALOHA is shown in Figure 4, in which the 


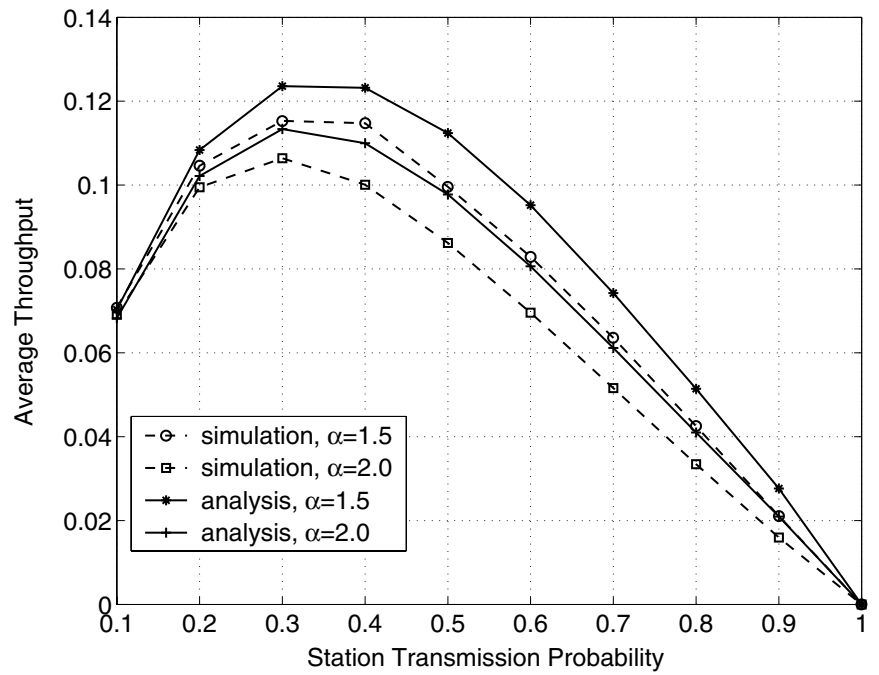

Fig. 4. Impact of capture ratio on the average throughput for capture ALOHA schemes

capture ratios are 1.5 and 2.0, respectively. Evidently, when $\alpha$ becomes smaller, the throughput will become greater. It can be predicted that when $\alpha \rightarrow \infty$, the throughput of ALOHA with capture is the same as that of ALOHA without capture. The difference between the analysis and simulation results are due to the same reason as above.

Table II compares the performance of non-enhanced (i.e., the station transmission probability is fixed) and enhanced (i.e., each station transmits packets according to $p_{\text {opt }}$ ) slotted ALOHA schemes. Obviously, we can see that if each station can tune its packet transmission probability dynamically according to the "optimal transmission probability," the network throughput can be improved. In addition, we can see that the "optimal throughput" is even greater than the maximum value when the stations transmit packet with fixed probability from 0 to 1 . This is because the "optimal solution" for the instantaneous and average throughputs may be different, i.e., they may be different "maximum points." The reason for the difference between the simulation and analysis results has been explained above.

Clearly, the enhanced slotted ALOHA schemes perform better in multihop ad hoc networks, due to the dynamic tuning of the packet transmission probability according to the local network topology. That is, although the optimal packet transmission probability is obtained from the maximum instantaneous throughput analysis, the resulting average onehop throughput is fairly high too.

Generally, the assumptions in the throughput analysis is appropriate and can provide effective method to improve the network performance.

\section{CONCLUSION}

In this paper, we analyze the maximum throughputs of slotted ALOHA with and without capture. Different from prior research which always assumes that the packet transmission
TABLE II

Average Throughput of Non-ENHANCED AND ENHANCED SLOTTED ALOHA SCHEMES

\begin{tabular}{|l|c|c|c|c|}
\hline \multirow{2}{*}{} & \multicolumn{2}{|c|}{ non-capture } & \multicolumn{2}{c|}{ capture } \\
\cline { 2 - 5 } & $\begin{array}{c}\text { non-enhanced } \\
\text { (maximum) }\end{array}$ & enhanced & $\begin{array}{c}\text { non-enhanced } \\
\text { (maximum) }\end{array}$ & enhanced \\
\hline analysis & 0.1017 & 0.1132 & 0.1236 & 0.1379 \\
\hline simulation & 0.0954 & 0.1104 & 0.1153 & 0.1217 \\
\hline
\end{tabular}

probability is a constant value, we allow each station to control its local packet transmission probability dynamically.

The analysis and simulation results show that the network throughput is greatly affected by the node degrees, both for the non-capture and capture slotted ALOHA. The enhanced scheme can always achieve the maximum or nearly the maximum throughput through dynamically adjusting the packet transmission probability. In addition, the smaller the capture ratio $\alpha$, the greater the advantage of capture slotted ALOHA over non-capture slotted ALOHA.

\section{ACKNOWLEDGMENT}

This work is supported by the National Natural Science Foundation of China/Research Grants Council of HongKong Joint Research Scheme under Grant No. N_HKU 009/00 and No. 6001161943.

\section{REFERENCES}

[1] H. Takagi, L. Kleinrock, "Optimal Transmission Ranges for Randomly Distributed Packet Radio Terminals," IEEE Trans. on Commun., vol. 32, no. 3, pp. 246-257, Mar. 1984.

[2] T. C. Hou, V. O. K. Li, "Transmission Range Control in Multihop Packet Radio Networks," IEEE Trans. on Commun., vol. 34, no. 1, pp. 38-44, Jan. 1986.

[3] R. Nelson, L. Kleinrock, "The Spatial Capacity of a Slotted ALOHA Multihop Packet Radio Network with Capture," IEEE Trans. on Commun., vol. 32, no. 6, pp. 684-694, Jun. 1984.

[4] L. Kleinrock, F. A. Tobagi, "Packet Switching in Radio Channels: Part I-Carrier Sense Multiple-Access Modes and Their Throughput-Delay Characteristics," IEEE Trans. on Commun., vol. 23, no. 12, pp.14001416, Dec. 1975

[5] F. A. Tobagi, L. Kleinrock, "Packet Switching in Radio Channels: Part II-The Hidden Terminal Problem in Carrier Sense Multiple-Access and the Busy-Tone Solution," IEEE Trans. on Commun., vol. 23, no. 12, pp.1417-1433, Dec. 1975.

[6] T. K. Philips, S. S. Panwar, and A N. Tantawi, "Connectivity Properties of a Packet Radio Network Model," IEEE Trans. on Info., vol. 35, no. 5, pp. 1044-1047, Sep. 1989.

[7] Y. Cheng, T. G. Robertazzi, "Critical Connectivity Phenomena in Multihop Radio Models," IEEE Trans. on Commun., vol. 37, no. 7, pp. 770-777, Jul. 1989.

[8] R. R. Boorstyn, A. Kershenbaum, B. Maglaris, and V. Sahin, "Throughput Analysis in Multihop CSMA Packet Radio Networks," IEEE Trans. on Commun., vol. 35, no. 3, pp. 267-274, Mar. 1987.

[9] Y. Wang, J. J. Garcia-Luna-Aceves, "Performance of Collision Avoidance Protocols in Single-Channel Ad Hoc Networks," in Proc. IEEE ICNP, Paris, France, Nov. 2002.

[10] G. Bianchi, "Performance Analysis of the IEEE 802.11 Distributed Coordination Function," IEEE J. Select. Area Commun., vol. 18, no. 3 , pp. 535-547, Mar. 2000.

[11] F. Cali, M. Conti, and E. Gregori, "IEEE 802.11 Protocol: Disign and Performance Evaluation of an Adaptive Backoff Mechanism," IEEE J. Select. Area Commun., vol. 18, no. 9, pp. 1774-1786, Sep. 2000.

[12] F. Cali, M. Conti, and E. Gregori, "Dynamic Tuning of the IEEE 802.11 Protocol to Achieve a Theoretical Throughput Limit," IEEE/ACM Trans. on Networking, vol. 8, no. 6, pp. 785-799, Dec. 2000. 